\title{
30 Jahre Zeitschrift Verhaltenstherapie: Die Verhaltenstherapie als Motor der Professionalisierung der Psychotherapie
}

\author{
Winfried Rief $^{a}$ Stefan G. Hofmann ${ }^{b}$ \\ ${ }^{a}$ AG Klinische Psychologie und Psychotherapie, Universität Marburg, Marburg, Deutschland; \\ ${ }^{b}$ Clinical Psychology Program, Boston University, Boston, MA, USA
}

Wo wäre die Psychotherapie heute, wenn nicht die Verhaltenstherapie ihre spezifischen Impulse eingebracht hätte, stimuliert, herausgefordert und Innovation vorangetrieben hätte? Das ist kaum vorstellbar. Psychotherapie wurde als etwas definiert, dessen Behandlungserfolg messbar ist. Nur wenn Behandlungserfolg messbar ist, kann geprüft werden, ob diese Behandlungen auch die Zielsetzungen von Kostenträgern, Patienten, Angehörigen und der Gesellschaft erfüllen. Damit wurde die Voraussetzung geschaffen, dass auch wissenschaftliche Studiendesigns entwickelt werden konnten, um sowohl die Effektivität sowie die verschiedenen Prozesse der Behandlung durch Psychotherapie zu evaluieren. Randomisierte klinische Studien (randomized clinical trials) wurden mit psychotherapeutischen Behandlungsansätzen durchgeführt und kontinuierlich weiterentwickelt. Manches Studiendesign, manche empirische Arbeit aus den 70er oder 80er Jahren hat damals hohen Stolz wegen der Innovationskraft ausgelöst, würde jedoch vermutlich heute vor Kritikern kaum mehr bestehen können. Die Qualitätsschraube der Ansprüche an wissenschaftliche Studien steigt kontinuierlich nach oben, und die Verhaltenstherapie hält Schritt. Gerade vor dem Hintergrund der Themen Replikationskrise und offene Wissenschaft [Shrout und Rodgers, 2018] ist auch nicht zu erwarten, dass sich die zunehmenden Anforderungen an Qualitätsmerkmale von Psychotherapiestudien in den nächsten Jahren oder Jahrzehnten verlangsamen würde.

Allerdings ist auch zu erwarten, dass sich die Studiendesigns und wissenschaftlichen Herangehensweisen an die Psychotherapie verbreitern werden. Zusätzlich zu

den experimentellen Ansätzen, wie sie sich in den randomisierten klinischen Studien zeigen, wächst die Relevanz anderer und neuer Studiendesigns (z.B. $N=1$ Studien, Mixed-Methods-Ansätze).

Durch die Forderung der Verhaltenstherapie, dass Psychotherapie wissenschaftlich evaluiert werden muss, wurde der wesentliche Impuls gesetzt, Psychotherapie als ein konkurrenzfähiges Angebot im Kontext verschiedener medizinischer und psychosozialer Behandlungsangebote zu sehen. Fragen der Konkurrenz oder Ergänzungsmöglichkeiten durch Psychopharmakotherapie konnten geprüft werden. Gerade unter der Perspektive von zeitlich befristeten Behandlungsangeboten konnte sich die Verhaltenstherapie positionieren als ein Behandlungsangebot, das lang über das Ende der Behandlung hinaus positive Wirkung zeigt - und sich damit deutlich von der Behandlung mit Psychopharmaka distanzieren kann [Voderholzer und Barton, 2016; Barton et al., 2018]. Ein breiter Siegeszug der Psychotherapie begann, der durch die ursprüngliche Forderung der Verhaltenstherapie nach Wissenschaftlichkeit stimuliert wurde. Wenn heute in fast allen Leitlinien zur Behandlung psychischer und psychosomatischer Erkrankungen Psychotherapie als "First-Line Treatment" angegeben wird und zum Teil die Vorteile bezüglich der Verläufe über die Behandlung hinaus betont werden, so basiert diese Empfehlung fast ausschließlich auf Therapiestudien zur Verhaltenstherapie.

Winfried Rief

AG Klinische Psychologie und Psychotherapie

Universität Marburg

Gutenbergstraße 18, DE-35032 Marburg (Deutschland)

rief@uni-marburg.de 
Aber auch ein anderer Impuls wurde durch die Verhaltenstherapie gesetzt, dessen Tragweite noch nicht vollständig genutzt und abgeschätzt werden kann: Es wurde dargelegt, dass sich eine Psychotherapieform nicht aus ihren eigenen theoretischen Überlegungen allein speisen darf, sondern sich auf breitere Grundlagenforschung beziehen muss. Jegliche Forschung zum Verhalten und Erleben von Menschen ist Grundlagenforschung für Psychotherapie und muss in der Weiterentwicklung von Konzepten und Behandlungsansätzen der Psychotherapie berücksichtigt werden [Holmes et al., 2018]. Durch diese neue Definition, dass Psychotherapie auch Grundlagenwissenschaften hat, verändert sich die komplette Psychotherapielandschaft. Psychotherapie ist nicht eine Summierung von abgegrenzten Theoriegebäuden einzelner Psychotherapieverfahren, sondern wird zu einer Wissenschaft, die den Bogen von Grundlagenforschung bis hin zur Versorgung spannt. Nebenbei war und ist dies auch eine Voraussetzung für die Psychotherapiereform, die die Psychotherapie-Ausbildung als einen eigenen Studiengang in Deutschland seit 2019 neu regelt.

An all diesen Entwicklungen war im deutschsprachigen Raum die Zeitschrift Verhaltenstherapie wesentlich beteiligt. Seit ihrer Gründung vor 30 Jahren hat sie sich schnell und systematisch zum angesehensten deutschsprachigen Publikationsorgan im Bereich Verhaltenstherapie entwickelt und auch international Anerkennung erhalten. Sie veröffentlichte nicht nur wichtige und vielzitierte wissenschaftliche Arbeiten, sondern setzte auch wesentliche neue Impulse und gab zukunftsgerichtete Innovationsvorschläge, aber auch Empfehlungen zu Qualitätskriterien und Behandlungsrichtlinien. Sie hat früh den Bogen gespannt zwischen Wissenschaft, Anwendungsfragen, manchmal Kasuistiken und darauf aufbauende Empfehlungen und Fragen der Versorgung. Die interessierte Leserin und der interessierte Leser finden aber auch Übersichtsarbeiten, um kondensiert ein "Up to date" zu einem bestimmten Thema zu bekommen. Dabei wurde immer eine gute Balance zwischen "innovativem Stimulieren" und "Bewährtes bewahren" angestrebt.

Die Psychotherapie allgemein und die Verhaltenstherapie im Spezifischen ist mitten in dem Prozess von dynamischen Weiterentwicklungen. Ein zunehmendes Verlassen von zwei, drei oder vier "Grundorientierungen" und hin zu mehr Pluralismus, Integration von Techniken aus verschiedenen Richtungen, Herausgreifen immer wieder neuer Prozesse und Behandlungsfoki werden eingeführt. Durch die zunehmende wissenschaftliche Fundierung solcher Innovationen sind diese auch nicht mehr so einfach wie früher als "unwissenschaftlich" zurückzuweisen, sondern fordern zur Integration und zum neuen Denken heraus. Gleichzeitig sind die traditionellen Werte und Impulse, die gerade die
Verhaltenstherapie eingebracht hat, alles andere als nicht mehr zeitgemäß. Mit der Wissenschaftlichkeit gibt es auch in dem neuen, erweiterten Feld der Verhaltenstherapie durchaus einige "Probleme". Immer noch werden neue Behandlungsansätze als neue Super-Heilsbringer vorgestellt, zum Teil mit ersten Studien mit eindeutiger Schlagseite unterfüttert, die uns an den alten Grundsatz "guter wissenschaftlicher Praxis" erinnern müssten: Wissenschaft dient nicht dazu, Werbematerial für subjektive Überzeugungen zu produzieren, sondern ist nur durch den eigenen kritischen Blick und kritische Bewertungen durch andere gerechtfertigt. Die kritischste Perspektive auf eine wissenschaftliche Arbeit muss der Autor selbst vornehmen - es gibt genügend Belege, dass dies im Bereich Psychotherapieforschung oftmals nicht gelingt [Munder et al., 2013]. Auch der von der Verhaltenstherapie aufgebrachte Fokus auf "beobachtbares Verhalten" ist heute so gültig wie früher, wenn auch selbstverständlich eine exklusive Eingrenzung auf beobachtbares Verhalten damit weder früher noch heute gemeint war. Aber wenn Behandlungserfolg sich nur in der Subjektivität des Behandlers oder des Patienten widerspiegelt, aber keine beobachtbaren Effekte für Patient, Familien und andere Angehörige, Arbeitgeber, Krankenkasse und Gesellschaft hat, dann kann dies nicht überzeugen. Last, not least: Der heute lautstark propagierte Ansatz der "Personalisierung von Psychotherapie" war von Anfang an ein Kennzeichen der Verhaltenstherapie. Durch eine individuelle Verhaltens- und Bedingungsanalyse sollte ein individueller Behandlungsplan entwickelt werden, der nur sekundär von der klassifizierten psychischen Störung abhing. Zwar hat die Verhaltenstherapie in den Folgejahren viel davon profitiert, Behandlungspakete für einzelne psychische Störungen vorzustellen und ihre Erfolge aufzuzeigen, trotzdem ist die Natur der verhaltenstherapeutischen Konzepte und Überlegungen von Anfang an die einer personalisierten Psychotherapie und nicht die einer "Psychotherapie für eine spezifisch klassifizierte psychische Krankheit".

Die Psychotherapie entwickelt sich entsprechend weiter und wird, wie unschwer zu erkennen ist, zunehmend die Verfahrensfamilien und ihre oft zu eng definierten Grenzen überwinden. Eine mögliche neue Denkalternative, die auch ein Gesamtkonzept für alle psychotherapeutischen Verfahren darstellen kann, ist die Fokussierung nicht auf eine grundlegende Theorie, sondern auf zentrale Mechanismen, Prozesse und Kompetenzen, wie sie auch die Grundlagenforschung definiert. Im angloamerikanischen Raum wird entsprechend das Konzept der prozessbasierten Therapie vorgestellt, in dem Psychotherapie nicht mehr als ein Gesamtpaket für eine bestimmte psychische Störung definiert wird, sondern als eine Kette von wissenschaftlich fundierten Prozessen [Hayes und Hofmann, 2018; Hofmann und Hayes, 2019].
Verhaltenstherapie 2021;31:5-7 DOI: $10.1159 / 000514718$
Rief/Hofmann 
Die wissenschaftliche Fundierung bezieht sich dabei nicht nur auf die Effektivitätsevaluation, sondern auch stark auf die dabei als zugrundeliegend angenommenen Mechanismen. Die Ausbildung von Psychotherapeutinnen und Psychotherapeuten wird damit weniger $\mathrm{zu}$ einer Ausbildung in der Theorie eines Verfahrens und mehr zu einer Ausbildung in Kompetenzen, die zur Veränderung der zentralen Mechanismen und Prozesse notwendig sind [Rief, 2021]. Dies trägt auch der Tatsache Rechnung, dass Psychotherapeutinnen und Psychotherapeuten zahlreiche Kompetenzen benötigen, die unabhängig von dem präferierten Verfahren sind [EACLIPT Task Force On "Competences of Clinical Psychologists", 2019].

Die Zeitschrift Verhaltenstherapie wird diese und zusätzliche Weiterentwicklungen nicht nur einfach begleiten, sondern weiterhin stimulieren, Impulse setzen, kritisch diskutieren, aber auch an die traditionellen, weiterhin gültigen Werte erinnern. Sie will eine Plattform sein, die im Zentrum dieser dynamischen Weiterentwicklungen im deutschsprachigen Bereich ist - und dabei auch selbst dynamisch bleibt. Psychotherapie ist eine sich voranschreitende Wissenschaft, und Verhaltenstherapie ist eine der treibenden Kräfte dieser Entwicklung.

Winfried Rief, Marburg

Stefan G. Hofmann, Boston

\section{Interessenskonflikt}

Die Autoren erklären, dass kein Interessenkonflikt besteht.

\section{Finanzielle Unterstützung für die hier dargestellte Arbeit}

Keine.

\section{Mitwirkung}

Beide Autoren trugen durch wesentliche inhaltliche Impulse zur Erstellung des Manuskriptes bei.

\section{Literatur}

Barton B, Schramm E, Voderholzer U. Short and long-term efficacy of psychotherapy compared to pharmacological treatment for depression. Z Psychiatr Psychol Psychother. 2018;66(1):19-28.

EACLIPT Task Force On "Competences of Clinical Psychologists”. Competences of Clinical Psychologists. Clin Psychol Eur. 2019;1(2): e35551.

Hayes SC, Hofmann SG. Process-based CBT: The science and core clinical competencies of cognitive behavioral therapy. New Harbinger Publications; 2018.
Hofmann SG, Hayes SC. The Future of Intervention Science: Process-Based Therapy. Clin Psychol Sci. 2019 Jan;7(1):37-50.

Holmes EA, Ghaderi A, Harmer CJ, Ramchandani PG, Cuijpers P, Morrison AP, et al. The Lancet Psychiatry Commission on psychological treatments research in tomorrow's science. Lancet Psychiatry. 2018 Mar;5(3):237-86.

Munder T, Brütsch O, Leonhart R, Gerger H, Barth J. Researcher allegiance in psychotherapy outcome research: an overview of reviews. Clin Psychol Rev. 2013 Jun;33(4):50111.
Rief W. Moving from tradition-based to competence-based psychotherapy. Evid Based Ment Health. 2021, DOI: 10.1136/ebmental-2020300219.

Shrout PE, Rodgers JL. Psychology, science, and knowledge construction: broadening perspectives from the replication crisis. Annu Rev Psychol. 2018 Jan;69(1):487-510.

Voderholzer U, Barton B. Short-term effects of Psychotherapy with no chronic depression: A systematic review of Studies compared with Pharmacotherapy. Verhaltenstherapie. 2016; 26(2):108-15. 